\title{
Double transposition and single branched TEVAR for total arch replacement in chronic dissection
}

\author{
Nimesh D. Desai, Grace Wang, Ashley Hoedt, Wilson Szeto, Joseph E. Bavaria \\ Division of Cardiovascular Surgery, Hospital of the University of Pennsylvania, Philadelphia, PA, USA \\ Correspondence to: Nimesh D. Desai, MD, PhD. Hospital of the University of Pennsylvania, Division of Cardiovascular Surgery, 3400 Spruce Street, \\ 6th Floor Silverstein, Philadelphia, PA 19104, USA. Email: nimesh.desai@uphs.upenn.edu.
}

Submitted Apr 23, 2018. Accepted for publication Apr 28, 2018.

doi: 10.21037/acs.2018.05.02

View this article at: http://dx.doi.org/10.21037/acs.2018.05.02

\section{Introduction}

Complex arch pathologies such as mid-arch aneurysms, mega-aortic syndrome and residual arch and distal descending aortic dissection traditionally require two step solutions to achieve a complete repair. This typically involves an open repair of the arch with either a traditional elephant trunk or frozen elephant trunk technique followed by open descending repair or stent grafting. More recently, hybrid arch repair has become available. Hybrid repairs require surgical reconstruction to bring the supra-aortic vessels more proximally off the ascending aorta and placement of a stent graft in the ascending aorta distal to their take-off. As for any endovascular stent graft procedure, the proximal and distal landing zones are crucial. Chronic residual arch and distal aortic dissection from an incomplete previous DeBakey I acute dissection repair is a uniquely difficult problem due to the re-do status of the patient and the complexity of the flap in the distal arch. However, they typically have surgical graft proximal landing zones which facilitate proximal seal.

As device technology has improved, emerging therapies for total aortic arch replacement have recently developed using primarily endovascular approaches for patients who required landing into Zone 0 . These include devices that have either a single branch and require a transposition or bypass to the left carotid and the left subclavian arteries or two branch devices that require bilateral carotid cut down, carotid-carotid bypass in the neck and left carotidsubclavian bypass.

\section{Clinical vignette}

The case presented is that of a 59-year-old male with a previous DeBakey I dissection repair followed several years later by a redo aortic valve replacement for valvular regurgitation. He presented with a $7 \mathrm{~cm}$ chronically dissected arch and distal aorta with a large fenestration in the proximal descending aorta. We performed a supraaortic debranching and placed a single branched Gore Thoracic Branch Endoprosthesis (TBE) device into an old ascending aortic surgical graft. This case represents a firstin-man experience of using the Gore TBE device in Zone 0 with this double transposition technique (Figure 1).

\section{Surgical techniques}

\section{Preparation}

The procedure is performed with electroencephalogram (EEG) monitoring as well as near-infrared spectroscopy to monitor cerebral perfusion. A small upper sternal split is performed with the oscillating sternotomy saw. The incision maybe extended slightly toward the patient's left into the supraclavicular region to improve exposure of the left subclavian artery, although this is not always necessary. As the entire operation is performed on the supra-aortic vessels, it is not necessary to expose a significant portion of the aorta itself or enter the pericardium. This is particularly helpful in the reoperative situation.

\section{Exposition}

We start with the careful dissection of the entire innominate artery including its bifurcation and dissection of the proximal origins of the right subclavian artery and the right carotid artery. These are then controlled with vessel loops. 

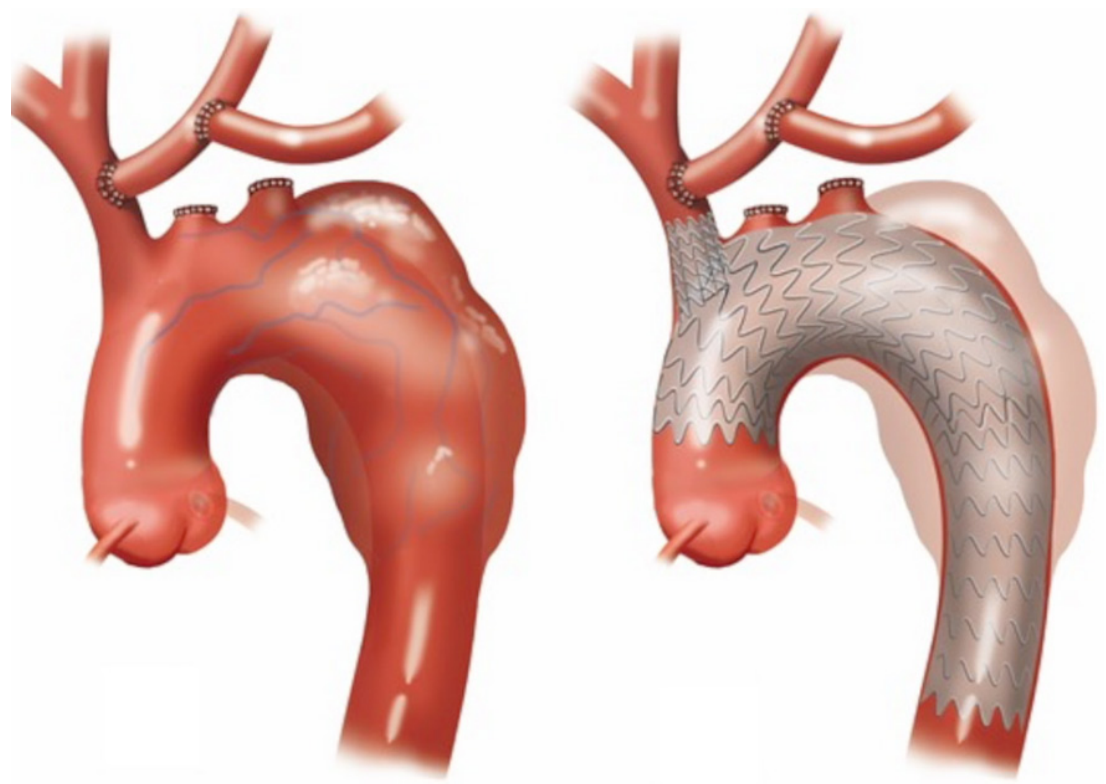

Figure 1 Double transposition and Zone 0 single branch TEVAR completion with Gore TBE for residual chronic arch and distal dissection. TBE, Thoracic Branch Endoprosthesis.

Next, the carotid is dissected from its origin off of the aortic arch all the way up into the neck. At least $5-7 \mathrm{~cm}$ of the artery should be mobilized as it has to be swung over to be anastomosed onto the distal innominate artery. The left subclavian artery at this level is typically directly behind the left carotid artery and we usually do not dissect it out until the left carotid artery has been disconnected from the arch and transposed onto the innominate artery. Heparin is given.

\section{Operation}

We then perform test clamping by placing a side-bite clamp on the distal portion of innominate artery just below its bifurcation. The side-bite clamp is used to maintain flow in the carotid artery on the right side. Right radial artery pressure is also monitored to ensure there is adequate perfusion pressure and that the side-bite clamp is not occlusive in the innominate artery. The left carotid is then clamped high up into the neck. Blood pressure is optimized to ensure higher perfusion pressures during this time. If there are no changes in cerebral saturation or the EEG, we then proceed with the transposition. To minimize ischemic time, the origin of the carotid is ligated at the level of the aortic arch using a TA 30 vascular load stapler. Distal to the staple line, the carotid is divided and fully mobilized so it can be slung up onto the innominate artery. The carotid has to be placed about $3 \mathrm{~cm}$ distal to the aortic origin of the innominate artery. This provides an appropriate landing zone for the side-branch graft that will go into the innominate artery.

A longitudinal incision is made on the medial leftward side of the innominate artery equivalent to the size of the left carotid artery. The left common carotid artery is then anastomosed in an end-to-side fashion to the innominate artery. Care is taken to flush these arteries of debris prior to removing all the clamps and reestablishing circulation. Once the carotid has been mobilized, the subclavian artery can be found directly posterior. This is also mobilized from its origin off of the aorta up to the vertebral branch. The subclavian is clamped distally. Again, a vascular load stapler is used to divide the subclavian origin off of the aorta. The subclavian is then fully mobilized and anastomosed to the underside of the left carotid artery. This completes the repair, as shown in Panel B of the figure. A drain is placed and brought through a separate stab incision. The sternum is re-approximated with two wires and the incision is closed. Typically, we have waited two to three days before performing the branched stent graft.

In this case, The TEVAR was performed using femoral access. A soft Bentson wire was placed in the true lumen via the right femoral artery and the position was verified with IVUS. This is exchanged for a double curved Lunderquist wire. A large bore Gore femoral sheath is placed for delivery 
of the aortic device. Contralateral femoral access is obtained for diagnostic angiography and a pig-tail catheter is placed in the ascending aorta. Right brachial access was also attained and a soft wire was passed into the descending aorta. After verification of the wire in the true lumen by IVUS, the brachial wire is snared from the Gore sheath and externalized. Using a quick-cross catheter, this is exchanged for an extra stiff wire. The aortic component of the Gore TBE graft is mounted on the Lunderquist wire in the main body lumen and the extra stiff wire in the side-branch portal lumen.

The graft is then advanced, avoiding wire wrap and maintaining rotational alignment between the side-branch portal and the greater curve of the arch. Once adequate positioning is achieved, the branch is deployed with the proximal component landing in the previous surgical ascending aortic graft and the portal adjacent to the innominate take-off. The side-branch sheath is then advanced over the extra-stiff wire and passed though the portal into the innominate artery. The sheath dilator is removed and the side-branch stent is advanced into the innominate. The sheath is retracted and the stent is deployed. The seal zones may be gently profile ballooned. The repair was extended with distal extensions (Gore cTAG) to approximately the level of the diaphragm. The patient had no significant complications and was discharged home with a completely thrombosed false lumen in the thorax.

\section{Comments}

At this time, there are no approved branched devices for the aortic arch in the United States. There is an emerging experience with these technologies within Europe and Asia, primarily in two-branch technologies. In the United States, a single-branch graft is currently being enrolled in a pivotal study after successfully completing an early feasibility study for Zone 0 and Zone 2 indications (1). Within the context of this early feasibility study, two techniques for modifying the cerebral circulation such that all of the supra-aortic flow arises from the innominate artery, thereby allowing for a TEVAR graft with a single branch to come across the entire arch and maintain perfusion to the head and upper body are employed. The first is a more standard right carotidto-left carotid bypass in the neck, usually retropharyngeal, followed with a left carotid-left subclavian bypass. This is a well described operation and in experienced hands has had good results. There are, however, concerns about graft thrombosis and, more importantly, swallowing difficulties and esophageal impingement $(2,3)$.
In an effort to avoid foreign graft material and maintain a more anatomic orientation of the head vessels, we developed a modification of an arch debranching-type operation previously described by Czerny et al. (4). This procedure involves a small upper sternal split and a direct left carotidto-innominate artery transposition and a left subclavian-toleft carotid transposition. The potential advantage of this procedure is that the supra-aortic vessels are maintained in an anatomic orientation and lie behind the sternum, eliminating the risk of esophageal complications and the procedure can usually be done with no graft material, eliminating the risk of thrombosis.

\section{Acknowledgements}

None.

\section{Footnote}

Conflicts of Interest: Drs. Bavaria, Szeto and Desai are investigators in the GORE TBE early feasibility trial. Drs. Bavaria and Desai are consultants to W. L. Gore and Associates. The other authors have no conflicts of interest to declare.

\section{References}

1. Patel HJ, Dake MD, Bavaria JE, et al. Branched Endovascular Therapy of the Distal Aortic Arch: Preliminary Results of the Feasibility Multicenter Trial of the Gore Thoracic Branch Endoprosthesis. Ann Thorac Surg 2016;102:1190-8.

2. Egaña JM, Sánchez J, Rodríguez V, et al. A technical improvement in retropharyngeal carotid reconstruction. J Vasc Surg 2012;56:542-4.

3. Ozsvath KJ, Roddy SP, Darling RC 3rd, et al. Carotidcarotid crossover bypass: is it a durable procedure? J Vasc Surg 2003;37:582-5.

4. Czerny M, Funovics M, Schoder M, et al. Transposition of the supra-aortic vessels before stent grafting the aortic arch and descending aorta. J Thorac Cardiovasc Surg 2013;145:S91-7.

Cite this article as: Desai ND, Wang G, Hoedt A, Szeto W, Bavaria JE. Double transposition and single branched TEVAR for total arch replacement in chronic dissection. Ann Cardiothorac Surg 2018;7(3):434-436. doi: 10.21037/ acs.2018.05.02 